\title{
1-Polarized pseudo-hexagons
}

\author{
Joseph A. Thas Hendrik Van Maldeghem*
}

\begin{abstract}
In this paper we continue our study begun in [4] aiming at characterizing the embedding of the split Cayley hexagons $\mathrm{H}(q)$, $q$ even, in $\mathrm{PG}(5, q)$ by intersection numbers with respect to their lines. We prove that, for $q \neq 3$, every pseudo-hexagon (i.e. a set $\mathcal{L}$ of lines of $\mathrm{PG}(5, q)$ with the properties that (1) every plane contains 0,1 or $q+1$ elements of $\mathcal{L}$, (2) every solid contains no more than $q^{2}+q+1$ and no less than $q+1$ elements of $\mathcal{L}$, and (3) every point of $\mathrm{PG}(5, q)$ is on $q+1$ members of $\mathcal{L}$ ) which is 1-polarized at some point $x$ (i.e., the lines of $\mathcal{L}$ through $x$ do not span $\operatorname{PG}(5, q)$ ) is either the line set of the standard embedding of $\mathrm{H}(q)$ in $\mathrm{PG}(5, q)$, or $q=2$ (in the latter case all pseudo-hexagons are classified in [4]).
\end{abstract}

Keywords: generalized hexagons, embedding

MSC 2000: 51E12, 51E20

\section{Introduction}

In the present paper, we continue our investigations begun in [4]. Let us recall briefly that the general aim is to characterize the standard embedding in $\mathrm{PG}(5, q)$ of the split Cayley hexagon $\mathrm{H}(q)$, $q$ even, by intersection numbers with subspaces. Roughly, since the points of $\mathrm{H}(q)$ are all the points of $\mathrm{PG}(5, q)$, we consider the intersections of subspaces with the line set of $\mathrm{H}(q)$. We also require that we deal with a tactical configuration, i.e., we assume that each point of the projective space is incident with exactly $q+1$ lines of our set. A similar characterization for the standard embedding of $\mathrm{H}(q)$ in $\mathrm{PG}(6, q)$ has been proved in [3].

\footnotetext{
${ }^{*}$ Both authors are partly supported by a Research Grant of the Fund for Scientific Research Flanders (FWO - Vlaanderen)
} 


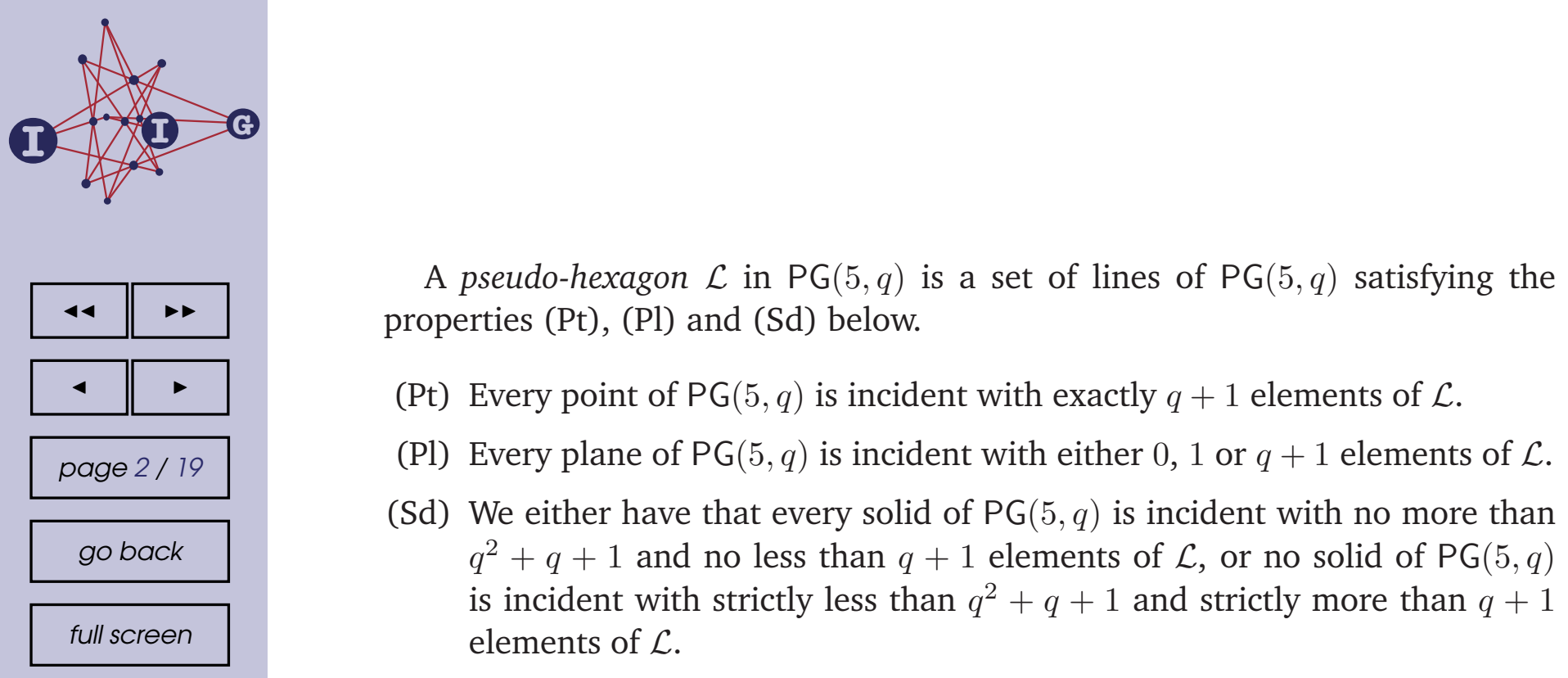

It is shown in [4] that a pseudo-hexagon also satisfies the following intersection properties.

$\left(\mathrm{Sd}^{\prime}\right)$ Every solid of PG $(5, q)$ is incident with either $q^{2}+q+1$ or $q+1$ elements of $\mathcal{L}$.

(Hp) Every hyperplane of PG $(5, q)$ is incident with exactly $q^{3}+q^{2}+q+1$ members of $\mathcal{L}$.

(To) The set $\mathcal{L}$ contains $q^{5}+q^{4}+q^{3}+q^{2}+q+1$ lines.

A pseudo-hexagon $\mathcal{L}$ with the additional property that for some point $x$, the members of $\mathcal{L}$ through $x$ are contained in a plane (hyperplane) will be called flat (1-polarized), and the point $x$ will also be called flat (1-polarized). The reason for not introducing such a notion for lines through a point to be contained in a solid is the following result proved in [4].

Fact 1.1 ([4]). If $\mathcal{L}$ is a pseudo-hexagon in $\mathrm{PG}(5, q), q \neq 2$, and for some point $x$ the members of $\mathcal{L}$ through $x$ are contained in a solid, then $\mathcal{L}$ is flat, all points of $\mathrm{PG}(5, q)$ are flat and $\mathcal{L}$ is the line set of a naturally embedded split Cayley hexagon $\mathrm{H}(q)$ in $\mathrm{PG}(5, q)$, with $q$ even. If $q=2$ and some point $x$ is flat, then we have the same conclusion. Conversely, the line set of every regularly embedded split Cayley hexagon $\mathrm{H}(q)$ in $\mathrm{PG}(5, q)$, q even, is a pseudo-hexagon for which all points are flat.

The last assertion of the previous theorem is of course the main motivation for studying pseudo-hexagons. Another motivation is the fact that also the line sets of some natural geometries related to a Singer cycle in $\mathrm{PG}(5, q)$ turn out to be pseudo-hexagons, as was also shown in [4], and these geometries were called Singer geometries. In the present paper, we improve on the above theorem by relaxing the condition on the point $x$, to $x$ being 1-polarized. This cannot be sharpened anymore as the examples related to the Singer cycle show. Of course, one would like to conjecture that the only pseudo-hexagons are either the Singer geometries or the line sets of naturally embedded split Cayley hexagons. The results of the present paper will contribute towards this conjecture. 



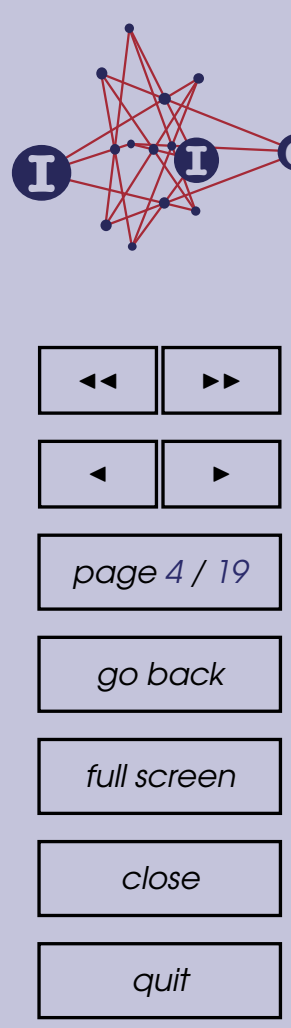

ing $(0,0,0,1,0,0,0)$ yields a representation of $\mathrm{H}(q)$ in $\mathrm{PG}(5, q)$. It is exactly this representation, up to projectivity, that we refer to as the naturally embedded split Cayley hexagon $\mathrm{H}(q)$ in $\mathrm{PG}(5, q)$. (Abstractly, an embedding of a point-line geometry $(\mathcal{P}, \mathcal{L}, \mathrm{I})$ in $\mathrm{PG}(n, q)$, for some $n$, is an injective mapping of $\mathcal{P}$ in the point set of $\mathrm{PG}(n, q)$ inducing an injective mapping from $\mathcal{L}$ into the line set of $\mathrm{PG}(n, q)$ and such that the image of $\mathcal{P}$ generates $\mathrm{PG}(n, q)$.)

We note that the above projection of $\mathrm{H}(q)$ as substructure of $\mathrm{Q}(6, q)$ induces a symplectic polarity $\rho$ in $\mathrm{PG}(5, q)$ with the property that all lines of $\mathrm{H}(q)$ in $\mathrm{PG}(5, q)$ are absolute lines with respect to $\rho$. The image of a line $L$ of $\mathrm{H}(q)$ under $\rho$ is an absolute solid $S$ which contains exactly all lines of $\mathrm{H}(q)$ that intersect $L$; hence $S$ contains $q^{2}+q+1$ lines of $\mathrm{H}(q)$ (see the first three paragraphs of Section 3 of [4]).

In the course of the proof of our Main Result, we will need to refer to many properties of pseudo-hexagons proved in [4]. One particularly beautiful and useful property is worth mentioning in this introduction and it is the following. If $\mathcal{L}$ is a pseudo-hexagon in $\operatorname{PG}(5, q)$, then the set of solids of $\operatorname{PG}(5, q)$ containing exactly $q^{2}+q+1$ members of $\mathcal{L}$ is a pseudo-hexagon in the dual of $\operatorname{PG}(5, q)$. This correspondence is more explicit if one considers the various types of subspaces of $\operatorname{PG}(5, q)$ with respect to the number of elements of $\mathcal{L}$ they contain, but it is also subtle: there is no duality involved of $\mathrm{PG}(5, q)$ (or at least, not necessarily). However, in the example above of the line set of the split Cayley hexagon $\mathrm{H}(q)$, this duality property is made explicit by the polarity $\rho$.

Finally we motivate the notion "1-polarized". In the literature, a polarized embedding of a geometry whose incidence graph has diameter $2 n$ is an embedding for which the lines at (graph-theoretical) distance at most $2 n-3$ from any given point is contained in a hyperplane. We generalize this as follows. For every natural number $i \geq 1$, and every geometry $\Omega$ of diameter $n \geq i+3$, we say that an embedding of $\Omega$ in some projective space is $i$-polarized if for every element (point or line) $x$ of $\Omega$ the set of lines at distance at most $i$ from $x$ is contained in a hyperplane. One can check that the case $i=n-3$ corresponds to the classical notion of polarized. Also, the restriction $i \leq n-3$ is necessary since for $i \geq n-2$ the definition would imply that the whole of $\Omega$ is contained in a hyperplane, contradicting the definition of embedding (which requires that the point set of $\Omega$ spans the projective space).

\section{Proof of the Main Result}

Standing Hypotheses. In this section, we assume that $\mathcal{L}$ is a set of lines of $\mathrm{PG}(5, q)$ satisfying the conditions (Pt), (Pl) and (Sd). Also, we assume that 










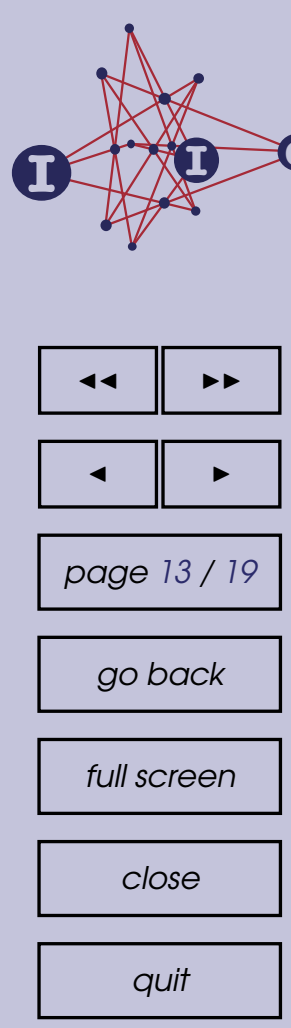

$2 q-3$ isolated lines meet one of these two lines (and $q-1$ of them meet $a \bar{a}$ ). Likewise, $q-1$ isolated lines meet $b \bar{b}$ and $q-2$ meet $b \bar{a}$. Moreover, since isolated lines cannot be contained in planes of Type I, every isolated line that meets $a \bar{a}$ also intersects $b \bar{b}$. We denote this set of isolated lines by $\mathcal{I}$; the other $q-2$ isolated lines are gathered in the set $\mathcal{I}^{\prime}$ and must all meet both of $a \bar{b}$ and $b \bar{a}$.

Now we consider an arbitrary point $c \in \lambda_{2}^{*}$.

First assume that $c \bar{c}$ intersects two lines $U$ and $U^{\prime}$ in $\mathcal{I}$. Then $L_{1}, L_{2}, U, U^{\prime}$ belong to a common regulus. Let $e \in \lambda_{2}^{*}$ and take the line $V$ containing $e$ and intersecting $L_{1}, L_{2}, U, U^{\prime}$. If $V \neq e \bar{e}$, then $V$ intersects exactly $\ell-1=1$ isolated line, a contradiction. Hence $V=e \bar{e}$ and so all $q+1$ lines $d \bar{d}$ form a regulus. Now assume that some line $d \bar{d}$ intersects $U^{\prime \prime}, U^{\prime \prime \prime} \in \mathcal{I}^{\prime}$, with $U^{\prime \prime} \neq U^{\prime \prime \prime}$. Then each of the lines containing a point of $\lambda_{1}$ and intersecting $U^{\prime \prime}, U^{\prime \prime \prime}$ is of the form $g \bar{g}$ (because it must meet at least two isolated lines). So these $q-1$ lines $g \bar{g}$ belong to a common regulus, which, by the above, also contains $a \bar{a}$ and $b \bar{b}$. But this contradicts the fact that $U^{\prime \prime}$ and $U^{\prime \prime \prime}$ belong to the opposite regulus. Hence $d \bar{d}$, with $d \in \lambda_{1}$, intersects at most one line of $\mathcal{I}^{\prime}$ and at least $q-2$ lines of $\mathcal{I}$. So at least $q$ lines of $\left\{L_{1}, L_{2}\right\} \cup \mathcal{I}$ belong to a common regulus. It follows that $\left\{L_{1}, L_{2}\right\} \cup \mathcal{I}$ is a regulus. All lines not of type $g \bar{g}$ joining a point of $\lambda_{1}$ to a point of $\lambda_{2}^{*}$ intersect exactly one line of $\mathcal{I}^{\prime}$.

We consider the bundle $\mathcal{B}$ of all quadrics containing the lines $a b, \bar{a} \bar{b}, a \bar{b}$ and $\bar{a} b$. The $q-2$ lines of $\mathcal{I}^{\prime}$ belong to $q-2$ distinct respective elements of $\mathcal{B}$. The three remaining members of $\mathcal{B}$ are the degenerate quadrics $\mathcal{Q}:=\langle a, b, \bar{b}\rangle \cup\langle\bar{a}, \bar{b}, b\rangle$ and $\mathcal{Q}^{\prime}:=\langle a, b, \bar{a}\rangle \cup\langle\bar{a}, \bar{b}, a\rangle$, and some quadric $\mathcal{H}$. Suppose now some point $x$ on a line of $\mathcal{I}$, with $x \notin a \bar{a} \cup b \bar{b}$, does not belong to $\mathcal{H}$. Then, since $x$ does clearly not belong to either $\mathcal{Q}$ nor $\mathcal{Q}^{\prime}$, it belongs to some quadric $\mathcal{Q}^{*}$ which contains a member $U$ of $\mathcal{I}^{\prime}$. But then $U$ meets the line through $x$ intersecting $L_{1}$ and $L_{2}$, and this line is of the form $g \bar{g}, g \in \lambda_{2}^{*}$, a contradiction. Hence all $(q-1)^{2}$ points on the lines of $\mathcal{I}$ not belonging to $a \bar{a}$ and $b \bar{b}$ belong to $\mathcal{H}$. Consequently $\left\{L_{1}, L_{2}\right\} \cup \mathcal{I}$ is a regulus of $\mathcal{H}$, and so the lines of $\mathcal{I}$ intersect $\bar{a} b$ and $a \bar{b}$, clearly a contradiction.

We conclude that $c \bar{c}$, with $c \in \lambda_{2}^{*}$, intersects all lines of $\mathcal{I}^{\prime}$. So the former $q-1$ lines belong to a common regulus, and the lines of $\mathcal{I}^{\prime}$ belong, together with $L_{1}$ and $L_{2}$, to the opposite regulus; both reguli belong to a hyperbolic quadric $\mathcal{H}^{\prime}$. On each line $c \bar{c}, c \in \lambda_{2}^{*}$, there is one point $a_{c}$ which belongs to some line of $\mathcal{I}$. These points $a_{c}, c \in \lambda_{2}^{*}$, belong to a common line $M$ which intersects $a \bar{b}$ and $\bar{a} b$, say in the points $u$ and $v$, respectively. It follows that the set of lines intersecting the three skew lines $L_{1}, L_{2}, M$ is precisely the set $\{a \bar{b}, \bar{a} b\} \cup \mathcal{I}$. Hence this set belongs to some quadric $\mathcal{H}$ which intersects $\mathcal{H}^{\prime}$ in the union of lines $a \bar{b} \cup \bar{a} b \cup M$.

Let $c \in \lambda_{2}^{*}$. The plane $\left\langle c, L_{1}\right\rangle$ contains $q-1$ lines of $\mathcal{L}$ on $c$. This plane intersects $\mathcal{H}$ in a nonsingular conic $C$. So $\left\langle c, L_{1}\right\rangle$ contains $q-1$ lines through $c$ 


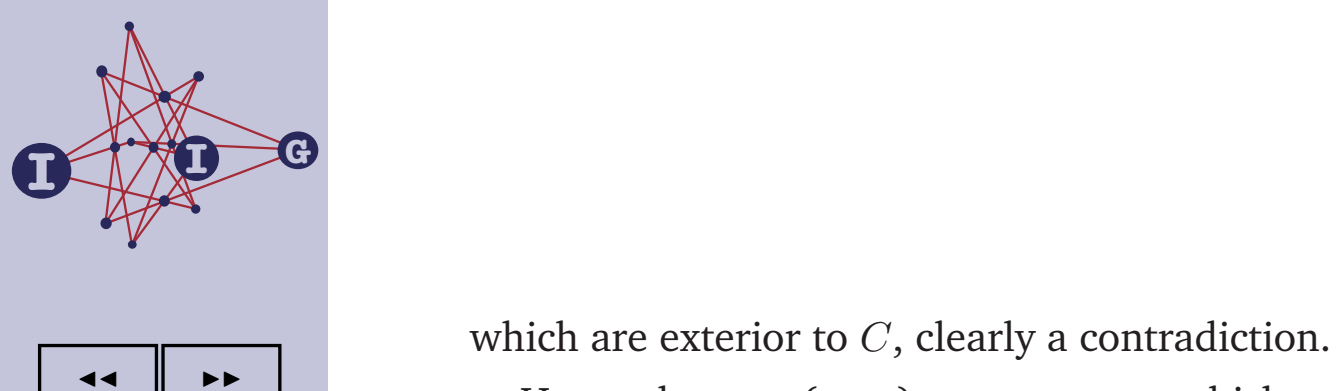

Hence the case (PAIR) cannot occur, which completes the proof of the lemma and of our Main Result.

\section{Addendum: The case (HALF) in dimension 3}

As is clear from the previous proofs, we have shown for $q \neq 3$ non-existence of pseudo-hexagons with a 1-polarized non-flat point just by proving that the structure induced in the solid $S$ cannot exist, except possibly in the case (HALF), where we used two such solids and their interaction. Let us call a line set in $\mathrm{PG}(3, q)$ consisting of $q^{2}+q+1$ lines meeting the properties of Lemma 2.5 a demi-system. One might wonder whether demi-systems in $\mathrm{PG}(3, q)$ exist at all. Of course, if one such system exists, then by our previous results, we have, with the notation of Lemma 2.5, $\ell_{1}=\ell_{2}=\frac{q+1}{2}$ (from which comes "demi" in the name). In fact, such structures exist, and we present a construction below. The motivation for this explicit construction is the following conjecture.

Conjecture 3.1. For each odd $q$ there is, up to projective equivalence, a unique demi-system in $\mathrm{PG}(3, q)$.

For the moment the only reason for this conjecture is curiosity. But in view of the beautiful properties that demi-systems enjoy, it is conceivable that they have other reasons to exist.

We end the present paper with the construction of a demi-system in $\operatorname{PG}(3, q)$, for all odd $q$.

Let $t \in \mathrm{GF}\left(q^{2}\right)$ have multiplicative order $q+1$. Then we can represent $\mathrm{PG}(3, q)$ as a subspace of $\mathrm{PG}\left(3, q^{2}\right)$ with the following point set:

$$
\begin{array}{r}
\mathcal{P}=\left\{\left(a(t+1) t^{k}, a(t+1), b t^{-h}, b t^{k+h}\right) \mid a, b \in \mathrm{GF}(q),(a, b) \neq(0,0),\right. \\
\text { and } h, k \in \mathbb{N}, 0 \leq h, k \leq q\} \\
\cup\left\{\left(a t^{k}, a, b t^{-h}, b t^{k+h-1}\right) \mid a, b \in \mathrm{GF}(q),(a, b) \neq(0,0),\right. \\
\text { and } h, k \in \mathbb{N}, 0 \leq h, k \leq q\} .
\end{array}
$$

In this setting, it is straightforward to check that the mapping

$$
\theta_{n}: \mathrm{PG}\left(3, q^{2}\right) \rightarrow \mathrm{PG}\left(3, q^{2}\right):(x, y, z, u) \mapsto\left(x t^{n}, y, z, u t^{n}\right)
$$

preserves $\mathrm{PG}(3, q)$ and hence defines a collineation of $\mathrm{PG}(3, q)$ fixing a spread $\mathcal{S}$ linewise. The partition induced on the point set by $\mathcal{S}$ is simply given by the orbits of the group $\Theta:=\left\{\theta_{n} \mid n \in \mathbb{Z}\right\}$ in $\mathrm{PG}(3, q)$. 
Now note that the inverse of $t$ coincides with its conjugate under the unique involutive automorphism of $\operatorname{GF}\left(q^{2}\right)$. Consequently expressions like $t^{n}+t^{-n}$ belong to $\mathrm{GF}(q)$, for $n \in \mathbb{Z}$.

We now first show a result on finite fields that makes our construction work. For $n \in \mathbb{Z} \backslash(q+1) \mathbb{Z}$, we put $f_{n}=\left(t^{n}-1\right)\left(t^{-n}-1\right) \in \mathrm{GF}(q)$.

Lemma 3.2. If $n$ is odd, then $f_{\ell} f_{\ell n}$ is a perfect square in $\operatorname{GF}(q)$, for all $\ell \in$ $\mathbb{Z} \backslash(q+1) \mathbb{Z}$.

full screen

close

quit

Proof. Indeed, one easily verifies that, putting $n=2 k+1$,

$$
f_{\ell} f_{\ell(2 k+1)}=\left(\left(t^{\ell(k+1)}+t^{-\ell(k+1)}\right)-\left(t^{\ell k}+t^{-\ell k}\right)\right)^{2} .
$$

Lemma 3.3. If $n \notin(q+1) \mathbb{Z}$ is even, then $f_{n} f_{2 n}$ is a perfect square in $\operatorname{GF}(q)$.

Proof. Indeed, one calculates that, putting $n=2 k$,

$$
f_{2 k} f_{4 k}=\left(\left(t^{3 k}+t^{-3 k}\right)-\left(t^{k}+t^{-k}\right)\right)^{2} .
$$

Lemma 3.4. If $f_{n}=f_{m}$, with $1 \leq n, m \leq q$, then $n=m$ or $n+m=q+1$.

Proof. From $f_{n}=f_{m}$ readily follows that $t^{n}+t^{-n}=t^{m}+t^{-m}=: T$. Hence $t^{n}, t^{-n}, t^{m}$ and $t^{-m}$ all satisfy the quadratic equation $X^{2}-T x+1=0$. Since this equation has at most two solutions over $\operatorname{GF}\left(q^{2}\right)$, the lemma follows easily.

Proposition 1. For all $n, m \in \mathbb{Z} \backslash(q+1) \mathbb{Z}$, we have that $f_{n} f_{m}$ is a perfect square in $\mathrm{GF}(q)$ if and only if $n+m$ is even.

Proof. Suppose first that $n+m$ is even. If $n$ is odd, then by Lemma 3.2, both $f_{1} f_{n}$ and $f_{1} f_{m}$ are squares in $\operatorname{GF}(q)$. Hence also $f_{1}^{2} f_{n} f_{m}$ is, and so also $f_{n} f_{m}$. If $n$ is even, put $n=2^{e} n^{\prime}$, with $n^{\prime}$ odd, and $m=2^{g} m^{\prime}$, with $m^{\prime}$ odd. Lemma 3.2 implies that $f_{n} f_{2^{e}}$ and $f_{m} f_{2^{g}}$ are squares in $\operatorname{GF}(q)$, while repeated use of Lemma 3.3 implies that $f_{2} f_{2^{e}}$ and $f_{2} f_{2^{g}}$ are squares in $\operatorname{GF}(q)$. Multiplying these four squares gives the desired result.

Now suppose that $n+m$ is odd and assume, by way of contradiction, that $f_{n} f_{m}$ is a square. Then either every $f_{i}, 1 \leq i \leq q$, is a square, or every such $f_{i}$ is a non-square in $\operatorname{GF}(q)$ (use the previous paragraph to see this). Lemma 3.4 implies that we obtain, in such a way, exactly $\frac{q+1}{2}$ non-zero squares or $\frac{q+1}{2}$ non-zero non-squares of $\mathrm{GF}(q)$, which both are contradictions.

We now construct a set $\mathcal{L}$ of $q^{2}+q+1$ lines. Therefore, we will set $\mathcal{L}=$ $\mathcal{L}_{0} \cup \mathcal{L}_{1} \cup \mathcal{L}_{2}$, where we define $\mathcal{L}_{i}, i=0,1,2$, below. 


\section{The set $\mathcal{L}_{0}$}

The elements of $\mathcal{L}_{0}$ are the $\left(\frac{q+1}{2}\right)^{2}$ lines joining a point $\left(t^{2 k+1}, 1,0,0\right)$ with a point $\left(0,0,1, t^{2 \ell}\right)$, with $0 \leq k, \ell<\frac{q+1}{2}$, together with the lines $L_{1}$ and $L_{2}$, where the line $L_{1}$ is the line consisting of the points $\left(t^{i}, 1,0,0\right), i \in \mathbb{Z}$, and the line $L_{2}$ is the line consisting of the points $\left(0,0,1, t^{j}\right), j \in \mathbb{Z}$.

Some more notation. The set $\lambda_{1}$ consists of the points $\left(t^{2 k+1}, 1,0,0\right)$, with $0 \leq k<\frac{q+1}{2}$, and the set $\lambda_{2}$ is the set of points $\left(0,0,1, t^{2 \ell}\right)$, with $0 \leq \ell<\frac{q+1}{2}$. Set $\lambda_{1}^{*}=L_{1} \backslash \lambda_{1}$ and $\lambda_{2}^{*}=L_{2} \backslash \lambda_{2}$.

In this notation, $\mathcal{P}_{0}=\left\{L_{1}, L_{2}\right\} \cup\left\{a_{1} a_{2} \mid a_{1} \in \lambda_{1}, a_{2} \in \lambda_{2}\right\}$.

In the sequel, if $a=\left(t^{i}, 1,0,0\right), i \in \mathbb{Z}$, we shall denote $\bar{a}=\left(0,0,1, t^{i}\right)$, and vice versa; i.e., if $\bar{a}=b$, then $\bar{b}=a$. Also, the plane spanned by $\left(t^{2 k+1}, 1,0,0\right)$ and $L_{2}$ will be denoted by $\pi_{2 k+1}, 0 \leq k<\frac{q+1}{2}$, and the plane spanned by $\left(0,0,1, t^{2 \ell}\right), 0 \leq \ell<\frac{q+1}{2}$, will be denoted by $\pi_{2 \ell}$. The planes $\pi_{i}, 0 \leq i \leq q$, will be the planes of Type I. We will also denote for short the point $\left(t^{i}, 1,0,0\right)$ by $x_{i}$, $0 \leq i \leq q$, and $\left(0,0,1, t^{j}\right)$ by $y_{j}, 0 \leq j \leq q$. Note that $\bar{x}_{i}=y_{i}$ and $\bar{y}_{j}=x_{j}$.

The set $\mathcal{L}_{1}$

A typical element of $\mathcal{L}_{1}$ is a line in $\pi_{2 k+1}$ containing $y_{2 k+1}$ and meeting the line $x_{2 k+1} y_{2 \ell}$ in the point $z_{k, \ell, A}$ with coordinates

$$
\left(t^{2 k+1}, 1, \frac{f_{2 k+1}}{\left(t^{2 \ell-2 k-1}-1\right)(t-1)} A, t^{2 \ell} \frac{f_{2 k+1}}{\left(t^{2 \ell-2 k-1}-1\right)(t-1)} A\right),
$$

where $A$ is a non-zero square in $\mathrm{GF}(q)$, or a line in $\pi_{2 \ell}$ containing $x_{2 \ell}$ and meeting the line $x_{2 k+1} y_{2 \ell}$ in the point $z_{k, \ell, B}$ with coordinates

$$
\left(t^{2 k+1}, 1, \frac{\left(t^{2 k-2 \ell+1}-1\right)(1-t)}{t f_{2 \ell-1}} B, t^{2 \ell} \frac{\left(t^{2 k-2 \ell+1}-1\right)(1-t)}{t f_{2 \ell-1}} B\right),
$$

where $B$ is a non-square in $\operatorname{GF}(q)$. One can check that the former elements of $\mathcal{L}_{1}$ are, for fixed $k$, independent of the choice of $\ell$, and the latter elements of $\mathcal{L}_{1}$ are, for fixed $\ell$, independent of the choice of $k$. Indeed, we check this claim in the first case. We have to show, for arbitrary integer $n$, that the unique $A^{\prime} \in \mathrm{GF}\left(q^{2}\right)$ making the determinant

$$
\left|\begin{array}{ccc}
1 & \frac{f_{2 k+1}}{\left(t^{2 \ell-2 k-1}-1\right)(t-1)} A & t^{2 \ell} \frac{f_{2 k+1}}{\left(t^{2 \ell-2 k-1}-1\right)(t-1)} A \\
1 & \frac{f_{2 k+1}}{\left(t^{2 n-2 k-1}-1\right)(t-1)} A^{\prime} & t^{2 n} \frac{f_{2 k+1}}{\left(t^{2 n-2 k-1}-1\right)(t-1)} A^{\prime} \\
0 & 1 & t^{2 k+1}
\end{array}\right|
$$





, Generalized hexagons and Singer geometries, Des. Codes Cryptogr. 47 (2008), 249-266.

[5] J. Tits, Sur la trialité et certains groupes qui s'en déduisent. Publ. Math. Inst. Hautes Étud. Sci. 2 (1959), 13-60.

[6] H. Van Maldeghem, Generalized Polygons, Monographs in Mathematics 93, Birkhäuser Verlag, Basel/Boston/Berlin, 1998.

full screen

close

quit

Joseph A. Thas

Ghent University, Pure Mathematics and Computer Algebra, Krijgslaan 281, S22, 9000 GENT, BELGIUM

e-mail: jat@cage.ugent.be

Hendrik Van Maldeghem

Ghent University, Pure Mathematics and Computer Algebra, KrijgslaAn 281, S22, 9000 GENT, BELGIUM

e-mail: hvm@cage.ugent.be 